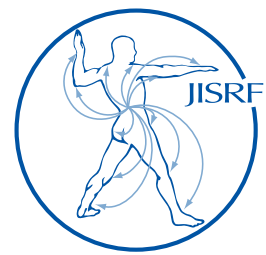

\title{
Oxford Partial Knee Replacement as a Gateway to Outpatient Arthroplasty "Lessons Learned Along the Journey"
}

\author{
Berend $M^{1}$
}

\begin{abstract}
The Oxford Partial Knee Replacement was approved for implantation in the US in 2004 after the surgeon completed an educational training requirement. Since then my knee practiced has expanded to over $50 \%$ partial knee. This experience coupled with refinement of surgical techniques, anesthesia protocols, and patient selection has facilitated the transformation to same day discharge for partial knee cases and has quickly transitioned to total hip, total knee, and selected revision surgeries. Patient selection has also expanded for outpatient joints and is now based on medical screening criteria and insurance access. Over a two-year period we have performed over 1,000 outpatient arthroplasty procedures with no readmissions for pain control. Overall readmission rate for all reasons was $2 \%$. Patient satisfaction scores were 98\% Great-Good for 2014-15. The combination of a partial knee replacement practice and an outpatient joint program brings the best VALUE to the patients, surgeons, and the arthroplasty system and represents the future of arthroplasty care.
\end{abstract}

Keywords: partial knee replacement; surgical technique; outpatient arthroplasty; patient satisfaction Level of Evidence: AAOS Therapeutic Level IV

\section{Introduction}

In 2004 the FDA approved the Oxford Partial Knee Replacement for implantation in the US after surgeons meet an educational training requirement. I was formerly biased against partial knee replacement believing it to be of limited value and indicated in only a small percentage of knees. [1] In the ensuing 12 years my knee practiced has been transformed to over $50 \%$ partial knee replacement. (Fig 1) This has largely been related to an improved understanding of the pathoanatomy of "anteromedial" osteoarthritis of the knee [2], comfort with the surgical technique $[3,4]$ and new instrumentation platform $[5,6]$, addition of lateral
1 Dr. Michael E. Berend, MD

Midwest Center For Joint Replacement, 6920 Gatwick Dr \#200, Indianapolis, IN 46241

Joint Replacement Surgeons of Indiana Research Foundation, 1199 Hadley Road, Mooresville, IN 46158

(Direct reprint requests to Michael Berend)

(c) 2016 Berend. All rights reserved. Reconstructive Review is a peer-reviewed, open-access orthopaedic OPEN 2 ACcEss
journal devoted to publishing papers in the area of reconstructive arthroplasty. Authors retain copyright and grant the journal right of first publication with the work. Reconstructive Review follows the Creative Commons AttributionNonCommercial CC BY-NC. This license allows anyone to download works, build upon the material, and share them with others for non-commercial purposes as long as they credit the senior author, Reconstructive Review, and the Joint Implant Surgery \& Research Foundation (JISRF). 


\section{Partial Knee Utilization by Year}

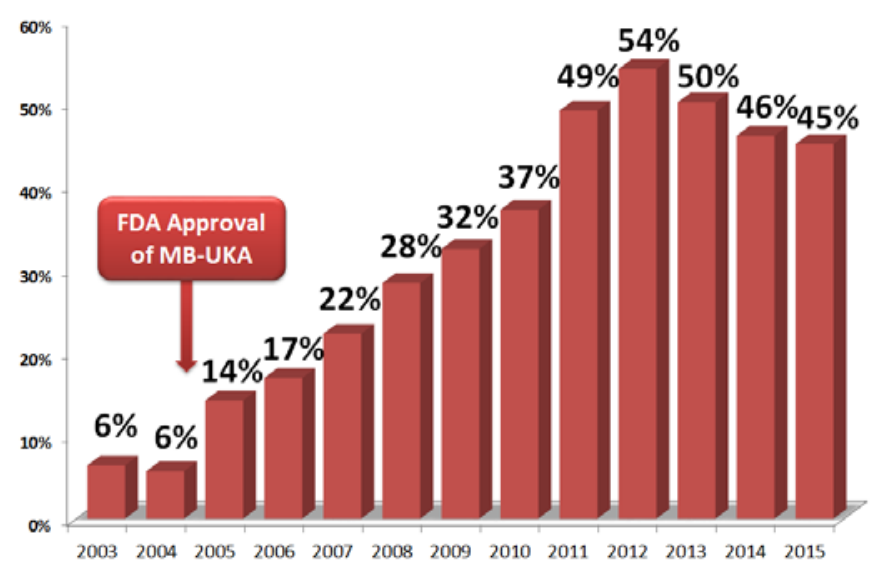

Figure 1. Percentage of Osteoarthritic knees receiving a partial compared to a total knee replacement at the Center for hip and Knee Surgery and Joint Implant surgeons from 2004 to 2015.

partial knee arthroplasty to my practice, and recognition of partial knee replacement being a lower morbidity procedure [7-9].

The hope of restoration of knee kinematics, decreasing polymer wear [10-12] through increased implant conformity, and lower polyethylene stresses are appealing with the Oxford mobile bearing device, which may improve longterm implant performance. Importantly however, mobile bearing UKA does not improve our indications, patient selection, or surgeon performance and are critical elements of UKA clinical success and survivorship. This growth in a desire to retain normal knee ligaments and joint surfaces combined with refinement of surgical techniques, anesthesia protocols, and patient selection has facilitated the transformation to same day discharge [13-15] initially for partial knee replacement which quickly transitioned to total hip and knee and now selected revision knee replacement surgery. This paper is a summary of the thoughts and processes that led to this transformation to partial knee replacement and outpatient arthroplasty becoming the most common procedures in my practice.

\section{Methods}

Patient selection for Oxford mobile bearing partial knee replacement is based predominantly on the pathoanatomy of the disease in medial compartment of the knee. The indication for medial UKA is anteromedial osteoarthritis (AMOA), a clinical condition originally described by White et al. [2] This disease involves complete bone-onbone arthrosis medially on a weight bearing radiograph, a functionally intact ACL and MCL, and a correctible varus deformity demonstrated on a valgus stress radiograph with preservation of functional cartilage in the lateral compartment.

Outpatient eligibility is now based on medical screening criteria and insurance access [13-15]. Patient selection focuses on medical suitability and insurance coverage. Currently Medicare only has an outpatient code for partial knee replacement (CPT 27446) and does not have an approved code for primary TKA (CPT 27447) nor primary THA (CPT 27130) in the outpatient setting. Non-Medicare cases are assessed on a case-by-case basis. The medical screening begins with assessment of an acceptable cardiac history, smoking cessation, recognition of obstructive sleep apnea, hemoglobin greater than 12 , and stable medical conditions such as diabetes, hypertension, and thyroid conditions. This is done on a case-by-case basis in conjunction with our anesthesia team. Careful screening for benign prostatic hypertrophy $(\mathrm{BPH})$ in males and obstructive sleep apnea (OSA) not treated with CPAP are critical. We do not have an independent BMI cutoff. We believe smoking cessation is critical and it is encouraged.

Candidates should be able to function independently with walker. Each patient and their family or caregivers are invited to attend a pre-op center visit and PT Evaluation. During this they receive pre-op education \& equipment assessment, tour the facility, meet the care team, and better understand the family support expectations after discharge from the center while at home. We have not tracked how many patients are offered the outpatient program and choose an inpatient stay.

The anesthetic and multimodal pain control program has been previously reported. [13-15] It essentially involves preoperative medications, intraoperative pericapsular joint injections, and standardized postoperative meds minimizing parental narcotics whenever possible. Additional medications utilized throughout the short stay experience include Tranexemic acid, Tylenol, Toradol, Pepcid, and Decadron. Meticulous timed administration of these meds coordinating anesthesia and nursing care is critically important.

From April of 2013 to June of 2015 we performed 1,029 arthroplasty cases in our outpatient program. The procedures included 446 partial knee replacements, 309 total knee replacements, and 274 total hip replacements. $48 \%$ were male. Patients received a detailed survey of their experience with the program that included a patient satisfaction evaluation. Postoperative issues were tracked monthly with surgeon reported readmissions, reoperations, and management of complications. 


\section{Results}

Growth of partial knee replacement has grown steadily over the past 12 years and plateaued at approximately $50 \%$ of all osteoarthritic knees now receive a UKA vs. a TKA. The overall patient satisfaction for 2014 - 2015 was 98\% Great / Good. (Table 1). Overall readmissions were $2 \%$. (Table 2). The majority of these were for an outpatient manipulation of the knee under anesthesia following TKA 9/24 (0.7\%).

Table 1: Patient Satisfaction Scores for 2014-2015 with the outpatient surgical program.

\begin{tabular}{|c|c|c|c|c|c|}
\hline 2014 & 1st qtr & 2nd atr & 3rd atr & 4th qtr & Average \\
\hline GREAT & $87.7 \%$ & $88 \%$ & $82 \%$ & $81 \%$ & $85 \%$ \\
GOOD & $11.1 \%$ & $10 \%$ & $15 \%$ & $17 \%$ & $13.3 \%$ \\
OK & $0.9 \%$ & $1 \%$ & $2.2 \%$ & $1.5 \%$ & $1.4 \%$ \\
FAIR & $0.4 \%$ & $0.4 \%$ & $0.5 \%$ & $0.2 \%$ & $0.4 \%$ \\
POOR & $0.05 \%$ & $0.3 \%$ & $0.3 \%$ & $0.4 \%$ & $0.3 \%$ \\
\hline 2015 & 1 st qtr & 2nd qtr & 3rd qtr & 4th qtr & Average \\
\hline GREAT & $87.6 \%$ & $90 \%$ & $88 \%$ & $91.8 \%$ & $89.4 \%$ \\
GOOD & $11 \%$ & $8 \%$ & $10 \%$ & $6.4 \%$ & $8.9 \%$ \\
OK & $0.9 \%$ & $1 \%$ & $4 \%$ & $1.3 \%$ & $1.8 \%$ \\
FAIR & $0.4 \%$ & $0.30 \%$ & $0.2 \%$ & $0.3 \%$ & $0.3 \%$ \\
POOR & $0.06 \%$ & $0 \%$ & $0.2 \%$ & $0.2 \%$ & $0.1 \%$ \\
\hline
\end{tabular}

Table 2: Surgeon reported readmissions following outpatient joint replacement.

\begin{tabular}{|l|l|}
\hline Readmissions & $24(2 \%)$ \\
\hline Manipulation & $9(0.7 \%)$ \\
\hline Medical & $6(0.5 \%)$ \\
\hline Wound & $5(0.4 \%)$ \\
\hline VTE & $2(0.16 \%)$ \\
\hline Infection & $2(0.16 \%)$ \\
\hline Pain Control & None, $0 \%$ \\
\hline
\end{tabular}

\section{Discussion}

\section{Partial Knee Replacement Program}

The indications for UKA have changed significantly over the last decade in our practice $[16,17]$ and may be changing throughout the US for mobile bearing metal backed implants based on emerging data. Traditional or "Classical" indications [18] preclude the use of UKA in patients younger than 60 , heavier than $82 \mathrm{~kg}$ (or BMI greater than 32), patients with radiographic patellofemoral disease, and patients with pain that is not isolated to the medial side of the knee. Although absolutely no data exists to corroborate these contraindications, surgeons frequently cite Kozin and Scott [18] when deciding whom to offer UKA. A number of studies have been done demonstrating these criteria do not have a negative effect on the outcome of the Oxford PKA. Furthermore, newer consensus guidelines have been proposed based on the evolution in our understanding of partial knee replacement over the past 30 years describing our journey to broader indications for the Oxford partial knee replacement with excellent long term results and survivorship. [19-21]

This shift is apparent in our previous publications. We have previously reported on a retrospective analysis of applying the Kozzin and Scott criteria to our TKA database and found between 4-6\% of varus knees would be candidates for UKA. [1] Since that time we have utilized the indication of a more physiologic criteria of "anteromedial" osteoarthritis (AMOA) of the knee with an intact ACL [2]. Our prevalence of UKA has gone from $4-6 \%$ in 2004 to over $50 \%$ in 2016. What has changed? I believe a better recognition of the unique AMOA pathophysiology that was described by White and Goodfellow [2] many years ago for medial compartment OA has accelerated this change.

Further investigation of BMI and its effect on survival was performed looking a series from Berend et al combined with that of the Oxford group. 2586 consecutive UKA were studied and life-tables were constructed to evaluate the effect of BMI on survival. Survival in 764 UKA with BMI 30-35 was 94\%, in 310 UKA with BMI 36-40 survival was $95 \%$, and in 209 knees with BMI >40 survival was $98 \%$. No statistical difference was seen between any BMI group ( $\mathrm{P}>0.05)$. [17]

Beard et al $[23,24]$ examined the role of patellofemoral disease and anterior knee pain on the outcomes of Oxford partial knee replacement. Berend, et al [17] sought to evaluate this in a US population. In this study standardized radiographs from 626 Knees evaluated the influence of the status of the patellofemoral joint on the outcome of the Oxford partial knee. in which a mobile bearing medial UKA was implanted were reviewed by an evaluator blinded to patient outcome or revision status. The evaluator recorded the pre-operative state of the patellofemoral joint using the Altman Classification. The Altman classification grades the patellofemoral joint medially and laterally for sclerosis, osteophytes, and joint space narrowing with a score of 0-4, with 4 representing severe, erosive bone-on-bone disease. Log-rank and Kaplan-Meier analysis were used to evaluate survivorship between knees with significant preoperative patellofemoral disease and those without. Within this subset of 626 knees, there were 17 revisions at up to 6 years (97.2\% survival). In only 384 knees $(61.3 \%)$ was 
the patellofemoral joint normal or Altman 0-1. Survival in this normal PFJ cohort was $93.8 \%$. In 242 knees, or $37.8 \%$ of cases, with pre-operative PFJ disease (Altman 2-4) the predicted survival was $97.9 \%$. Of these, 92 knees $(15 \%)$ had significant disease (Altman 3 or greater) and the survival was $97.0 \%$.

Perhaps most convincing, however, is the stark difference in morbidity and mortality associated with UKA when compared to TKA. Bergeson et al [7], evaluated 1,000 consecutive UKA for 90 day perioperative morbidity and mortality. There were no deaths $(0.0 \%)$, one DVT $(0.1 \%)$, and one deep infection $(0.1 \%)$. Five patients required a transfusion $(0.5 \%)$ and 7 patients had a cardiac complication including CHF, arrhythmia, or myocardial infarction $(0.7 \%)$. Thus the early morbidity associated with mobile bearing UKA warrants that this procedure be defined as truly minimally invasive, in contradistinction from TKA. With such a low rate of perioperative complications, surgeons may be over-treating patients with TKA in knees with anteromedial OA who meet the expanded indications for UKA, putting them at undue risk. These liberal indications for UKA, based on the patho-anatomic condition of Anteromedial Osetoarthritis, appear to be a safe and accurate measure of candidacy for UKA. [22] Liddle et al confirmed these findings in recent study Lancet. [9]

Excellent clinical and survivorship results with $98 \%$ survival at 6 years are seen with liberal indications using this mobile bearing partial knee replacement $[16,25]$ have been reported by Berend, et al. Longer-term results have been reported by Price and Svard to be $91 \%$ at 16-years and no additional failures at 20-years (91\%) [19-21]. The high success and low incidence of perioperative complications make this an ideally suited operation with nearly no contraindications in patients with anteromedial OA of the knee. These factors have led to the development of an outpatient partial knee replacement program.

\section{Outpatient Program Development}

Perhaps the most significant developments in joint replacement surgery in the past decade have been in the area of multimodal pain management. This has reduced length of stay in the inpatient hospital environment opening the opportunity for cost savings and paved the way for outpatient joint replacement surgery in appropriately selected patients. The synergy and implementation of the knowledge gained over the past two decades of arthroplasty care make outpatient joint replacement possible and effective. $[1,2]$

Refinement of surgical techniques, anesthesia protocols, and patient selection has facilitated a transformation to same day discharge for arthroplasty care in our practice.
[13-15] This initially began in September of 2011 with selected Partial Knee Replacement (PKR) cases. The surgical procedures included in the outpatient program have expanded to include primary TKA (Total Knee Arthroplasty), primary THA (Total Hip Arthroplasty), and selected revision cases. The trend for early discharge has already happened for procedures formerly regarded as "inpatient" procedures such as upper extremity surgery, arthroscopy, ACL reconstruction, foot and ankle procedures, and rotator cuff repair. The outpatient program centers on the patient needs, family engagement, essentials of home recovery, preoperative education, efficient surgery, and a surgeon controlled environment with highly standardized care. This is a distinct shift in today's healthcare environment, which has seen the expansion of regulatory demands; focus on Electronic Health Record (EHR), and discussions of potential future value creation.

The hallmark of this program is meticulous protocol execution and surgeon directed care pathways. Preemptive pain control with oral anti-inflammatory agents, gabapentin, regional anesthetic blocks that preserve quad function for TKA (adductor canal block) and pericapsular long acting local anesthetics with the addition of injectable ketorolac and IV acetaminophen are key adjuncts. Over the past two years utilizing this type of program the majority of our partial knee replacement patients are now returning home the day of surgery. [13-15]

Concerns over readmission are appropriate. The rates of complications and readmissions in our series at $2 \%$ are less than reported by other authors for inpatient cohorts [26]. Interestingly we have had no readmissions for pain control since the programs inception. The majority of readmissions were for manipulation done as an outpatient with the remainder being known complications following inpatient or outpatient arthroplasty care and not unique to their outpatient care. The program centers on the patient, their family, home recovery, preoperative education, efficient surgery, and represents a shift in the paradigm of arthroplasty care. It can be highly beneficial to patients, surgeons, anesthesia, facility costs, and payors as arthroplasty procedures shift to the outpatient space. We believe this brings the best VALUE to the patients, surgeons, and the arthroplasty system.

Patient Satisfaction scores were outstanding with this program achieving $98 \%$ Great/Good for 2014-15. We believe this brings the best VALUE to the patients, surgeons, and the arthroplasty system and represents the future of arthroplasty care with future growth of both partial knee replacements and outpatient arthroplasty.

In summary, unicompartmental knee arthroplasty (UKA) has seen increasing interest due to better under- 
standing of indications, implant and instrumentation design, minimally invasive techniques, and improved reported outcomes. Survivorship with revision of any kind of Mobile Bearing UKA appears to rival that of total knee arthroplasty, despite more liberal indications than those traditionally used. Growth of partial knee replacement has set the stage for the development of a successful outpatient joint replacement program for patients and surgeons alike.

\section{Disclosure}

The author declares that there is no conflict of interest regarding the publication of this paper. For full disclosures refer to last page of this journal.

\section{References}

1. Ritter MA, Faris PM, Thong AE, Davis KE, Meding JB, Berend ME. Intra-operative findings in varus osteoarthritis of the knee. An analysis of pre-operative alignment in potential candidates for unicompartmental arthroplasty. J Bone Joint Surg Br. 2004 Jan;86(1):43-7.

2. White SH, Ludkowski PF, Goodfellow JW. Anteromedial osteoarthritis of the knee. J Bone Joint Surg Br. 1991 Jul;73(4):582-6.

3. Clarius M, Hauck C, Seeger JB, James A, Murray DW, Aldinger PR. Pulsed lavage reduces the incidence of radiolucent lines under the tibial tray of Oxford unicompartmental knee arthroplasty: pulsed lavage versus syringe lavage. Int Orthop. 2009 Dec;33(6):1585-90. Epub 2009 Feb 14.

4. Pandit H, Jenkins C, Beard DJ, Gallagher J, Price AJ, Dodd CA, Goodfellow JW, Murray DW. Cementless Oxford unicompartmental knee replacement shows reduced radiolucency at one year. J Bone Joint Surg Br. 2009 Feb;91(2):185-9.

5. Koh IJ, Kim JH, Jang SW, Kim MS, Kim C, In Y.Are the Oxford(®) medial unicompartmental knee arthroplasty new instruments reducing the bearing dislocation risk while improving components relationships? A case control study. Orthop Traumatol Surg Res. 2016 Apr;102(2):183-7.

6. Hurst JM1, Berend KR2, Adams JB1, Lombardi AV Jr. Radiographic comparison of mobile-bearing partial knee single-peg versus twin-peg design. J Arthroplasty. 2015 Mar;30(3):475-8. doi: 10.1016/j.arth.2014.10.015. Epub 2014 Oct 23.

7. Bergeson AG, Berend KR, Lombardi AV Jr, Hurst JM, Morris MJ, Sneller MA. Medial mobile bearing unicompartmental knee arthroplasty: early survivorship and analysis of failures in 1000 consecutive cases. J Arthroplasty. 2013 Oct;28(9 Suppl):172-5.

8. Brown NM, Sheth NP, Davis K, Berend ME, Lombardi AV, Berend KR, Della Valle CJ. Total knee arthroplasty has higher postoperative morbidity than unicompartmental knee arthroplasty: a multicenter analysis. J Arthroplasty. 2012 Sep;27(8 Suppl):86-90.
9. Liddle AD, Judge A, Pandit H, Murray DW.Adverse outcomes after total and unicompartmental knee replacement in 101,330 matched patients: a study of data from the National Joint Registry for England and Wales. Lancet. 2014 Oct 18;384(9952):1437-45.

10. McGovern TF, Ammeen DJ, Collier JP, Currier BH, Engh GA. Rapid polyethylene failure of unicondylar tibial components sterilized with gamma irradiation in air and implanted after a long shelf life. J Bone Joint Surg Am. 2002 Jun;84$\mathrm{A}(6): 901-6$.

11. Kendrick BJ, Longino D, Pandit H, Svard U, Gill HS, Dodd CA, Murray DW, Price AJ. Polyethylene wear in Oxford unicompartmental knee replacement: a retrieval study of 47 bearings. J Bone Joint Surg Br. 2010 Mar;92(3):367-73.

12. Psychoyios V, Crawford RW, O'Connor JJ, Murray DW. Wear of congruent meniscal bearings in unicompartmental knee arthroplasty: a retrieval study of 16 specimens. J Bone Joint Surg Br. 1998 Nov;80(6):976-82.

13. Lombardi AV Jr, Barrington JW, Berend KR, Berend ME, Dorr LD, Hamilton W, Hurst JM, Morris MJ, Scuderi GR. Outpatient Arthroplasty is Here Now. Instr Course Lect. 2016;65:531-46.

14. Berend ME, Berend KR, Lombardi AV Jr. Advances in pain management: game changers in knee arthroplasty. Bone Joint J. 2014 Nov;96-B(11 Supple A):7-9.

15. Berend, ME, Lackey, WG, Carter, JL. Outpatient TJA: "Drive-Thru" Surgery Affirms, JBJS-B. CCJR proceedings. 2016.

16. Berend KR, Lombardi, Jr. AV, Hurst JM, Morris M, Indications for UKA, Is there any science, AAOS OLC presentation, Oct 2010.

17. Berend KR, Lombardi AV Jr, Adams JB.: Obesity, young age, patellofemoral disease, and anterior knee pain: identifying the unicondylar arthroplasty patient in the United States. Orthopedics. 2007 May;30(5 Suppl): 19-23.

18. Kozinn SC, Scott R. Unicondylar Knee Arthroplsty. JBJS 1989, 71Am; 145-150.

19. Price AJ, Waite JC, Svard U.: Long-term clinical results of the medial Oxford unicompartmental knee arthroplasty. Clin Orthop Relat Res. 2005 Jun;(435):171-80.

20. Price AJ, Svard U. A second decade lifetable survival analysis of the Oxford unicompartmental knee arthroplasty. Clin Orthop Relat Res 2010 Aug 13 (epub ahead of print).

21. Svärd UC, Price AJ. Oxford medial unicompartmental knee arthroplasty. A survival analysis of an independent series. J Bone Joint Surg Br. 2001 Mar;83(2):191-4.

22. Berend KR, Berend ME, Dalury DF, Argenson JN, Dodd CA, Scott RD. Consensus Statement on Indications and Contraindications for Medial Unicompartmental Knee Arthroplasty. J Surg Orthop Adv. 2015 Winter;24(4):252-6.

23. Beard DJ, Pandit H, Ostlere S, Jenkins C, Dodd CA, Murray DW.Pre-operative clinical and radiological assessment of the patellofemoral joint in unicompartmental knee replacement and its influence on outcome. J Bone Joint Surg Br. 2007 Dec;89(12):1602-7.

24. Beard DJ, Pandit H, Gill HS, Hollinghurst D, Dodd CA, Murray DW. The influence of the presence and severity of pre-existing patellofemoral degenerative changes on the outcome of the Oxford medial unicompartmental knee replacement. J Bone Joint Surg Br. 2007 Dec;89(12):1597-601.

25. Berend KR, Lombardi AV Jr, Mallory TH, Adams JB, Groseth KL.: Early failure of minimally invasive unicompartmental knee arthroplasty is associated with obesity. Clin Orthop Relat Res. 2005 Nov; 440: 60-6.

26. Edwards, et al. Avoiding Readmsisions - Support Systems Required after Discharge to Continue Rapid Recovery. J Arthoplasty 2015. 